\title{
High electron mobility and large magnetoresistance in the half-Heusler semimetal LuPtBi
}

\author{
Zhipeng Hou, ${ }^{1,2}$ Wenhong Wang,,${ }^{1, *}$ Guizhou Xu, ${ }^{1}$ Xiaoming Zhang, ${ }^{1}$ Zhiyang Wei, ${ }^{1}$ Shipeng Shen, ${ }^{1}$ Enke Liu, ${ }^{1}$ Yuan Yao, ${ }^{1}$ \\ Yisheng Chai, ${ }^{1}$ Young Sun, ${ }^{1}$ Xuekui Xi, ${ }^{1}$ Wenquan Wang, ${ }^{2}$ Zhongyuan Liu, ${ }^{3}$ Guangheng Wu, ${ }^{1}$ and Xi-xiang Zhang ${ }^{4}$ \\ ${ }^{1}$ State Key Laboratory for Magnetism, Beijing National Laboratory for Condensed Matter Physics, Institute of Physics, \\ Chinese Academy of Sciences, Beijing 100190, China \\ ${ }^{2}$ College of Physics, Jilin University, Changchun 130023, China \\ ${ }^{3}$ State Key Laboratory of Metastable Material Sciences and Technology, Yanshan University, Qinhuangdao 066004, China \\ ${ }^{4}$ Physical Science and Engineering, King Abdullah University of Science and Technology (KAUST), Thuwal 23955-6900, Saudi Arabia
}

(Received 23 March 2015; revised manuscript received 22 November 2015; published 18 December 2015)

\begin{abstract}
Materials with high carrier mobility showing large magnetoresistance (MR) have recently received much attention because of potential applications in future high-performance magnetoelectric devices. Here, we report on an electron-hole-compensated half-Heusler semimetal LuPtBi that exhibits an extremely high electron mobility of up to $79000 \mathrm{~cm}^{2} / \mathrm{V} \mathrm{s}$ with a nonsaturating positive MR as large as $3200 \%$ at $2 \mathrm{~K}$. Remarkably, the mobility at $300 \mathrm{~K}$ is found to exceed $10500 \mathrm{~cm}^{2} / \mathrm{V} \mathrm{s}$, which is among the highest values reported in three-dimensional bulk materials thus far. The clean Shubnikov-de Haas quantum oscillation observed at low temperatures and the first-principles calculations together indicate that the high electron mobility is due to a rather small effective carrier mass caused by the distinctive band structure of the crystal. Our findings provide a different approach for finding large, high-mobility MR materials by designing an appropriate Fermi surface topology starting from simple electron-hole-compensated semimetals.
\end{abstract}

DOI: 10.1103/PhysRevB.92.235134

PACS number(s): 75.47.-m, 71.18.+y, 71.55.Ak, 74.25.Jb

\section{INTRODUCTION}

The search for new materials with both high charge-carrier mobility and magnetoresistance (MR) is an important topic of research in condensed matter physics and for various device applications. In contrast to the large negative MR often observed in magnetic materials [1-6], several nonmagnetic material systems, ranging from narrow-gap semiconductors [7-9] and zero-gap semimetals [10-13] to two-dimensional transition metal oxides [14] and topological insulators [15,16], have recently been discovered to show a large nonsaturating positive MR. Attempts to explain this high MR include a classical MR effect based on a long transport mean free path due to mobility distribution [17] and the quantum effect with a linearly dispersing band structure [18]. Because materials with high MR and carrier mobility show promising applications for future high-performance spintronics devices, more investigation into the mechanism is warranted.

Compensated semimetals have approximately the same number of holes as electrons (a balanced hole-electron resonance condition) and thus a zero band gap at the Fermi surface. For these reasons, they are expected to be an ideal platform for exploiting a high positive MR; well-known examples include $\mathrm{Bi}$ and graphite $[10,11]$. Recently, the compensated semimetal $\mathrm{WTe}_{2}$ was shown to have a nonsaturating positive MR of up to $450000 \%$ at low temperatures [19], which would make it suitable for investigating the unusual transport properties and carrier scattering mechanisms of compensated semimetals. However, layered $\mathrm{WTe}_{2}$ loses its advantage at higher temperatures where its positive MR is reduced significantly due to the dramatic change in the Fermi surface, making it impractical for use in spintronics devices because they operate over a wide temperature range [20].

${ }^{*}$ Corresponding author: wenhong.wang@iphy.ac.cn
In parallel, many ternary half-Heusler compounds with 18 valence electrons that are naturally semimetals with tunable structural and electronic properties have been well studied for their multifunctional properties and their potential application as thermoelectric materials [21,22]. Recently, studies have focused on some heavy half-Heusler compounds composed of the rare earth elements $\mathrm{Ln}(\mathrm{Ln}=\mathrm{Sc}, \mathrm{Y}, \mathrm{La}, \mathrm{Lu})$ such as $\mathrm{LnPtSb}$ and LnPtBi. These compounds are of interest because they feature a zero-gap topologically nontrivial band inversion in the presence of spin-orbit coupling and may exhibit unprecedented features, such as high carrier mobility and MR when subjected to a magnetic field $H$ [23-28]. Despite considerable efforts, the highest carrier mobility observed in half-Heusler semimetals, like LaPtBi and YPtBi, is limited to $\sim 4000 \mathrm{~cm}^{2} / \mathrm{V} \mathrm{s}$ at room temperature (RT) [26]. Here, in this paper, we report an electron-hole-compensated half-Heusler semimetal LuPtBi with an electron mobility as high as $79000 \mathrm{~cm}^{2} / \mathrm{V} \mathrm{s}$ at $2 \mathrm{~K}$. Remarkably, the mobility at $300 \mathrm{~K}$ is found to exceed $10500 \mathrm{~cm}^{2} / \mathrm{V} \mathrm{s}$, which is comparable to the three-dimensional (3D) Dirac semimetal $\mathrm{Cd}_{3} \mathrm{As}_{2}[13,29]$. Note that the LuPtBi crystal MR increases from $260 \%$ at RT to $3200 \%$ at $2 \mathrm{~K}$. The combined ultrahigh mobility and MR across a wide temperature range make the compensated semimetal LuPtBi and related half-Heusler compounds potential material candidates for application to future spintronics devices.

\section{EXPERIMENTAL METHODS AND STRUCTURE DETERMINATION}

Single LuPtBi crystals were grown by a Bi-rich self-flux method. For the growth of LuPtBi crystals, the high-purity Lu (ingot, 99.99\% Pt (ingot, 99.99\%), and Bi (ingot, 99.99\%) starting materials were mixed together in a molar ratio of $1: 1: 10$, and afterward the mixture was placed in an alumina crucible. This process was performed in an argon-filled glove box, where the oxygen and humidity content were less than 
(a)

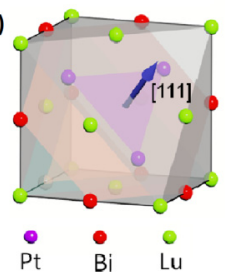

(b)

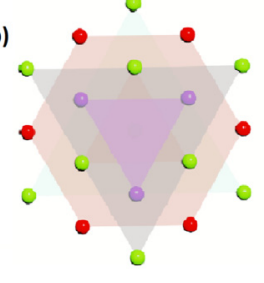

(c)

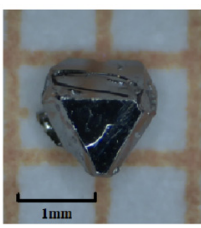

(d)

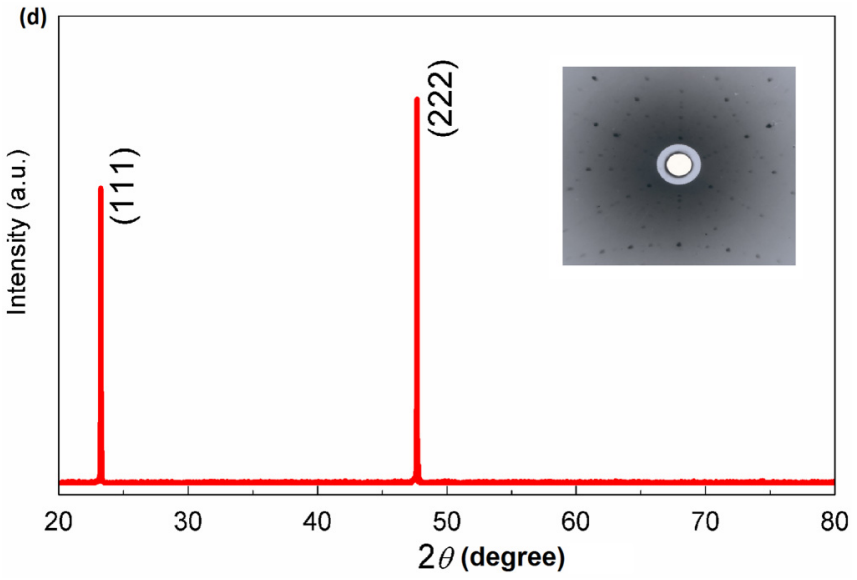

FIG. 1. (Color online) (a) The crystal structure of LuPtBi. The blue arrow shows the normal of the natural cleavage plane along the (111) surface. (b) The projected view of the lattice along the [111] direction, displaying Lu, Pt, and Bi stacked layers. (c) Photographs of synthesized single-crystal LuPtBi placed on a millimeter grid. One division represents $1 \mathrm{~mm}$ in the diagram; crystals were grown to $1.5 \times 1.0 \times 0.8 \mathrm{~mm}^{3}$, with mirrorlike surfaces and were robust in air. (d) XRD patterns with the $\mathrm{x}$ rays along the perpendicular direction of the hexagonal surfaces are presented.

$0.5 \mathrm{ppm}$. The whole assembly was sealed inside a tantalum tube under Ar gas, followed by sealing in an evacuated quartz tube. Crystal growth was performed in a furnace by heating the tube from room temperature to $1150^{\circ} \mathrm{C}$ over a period of $15 \mathrm{~h}$, maintained for $24 \mathrm{~h}$, and then slowly cooled to $650^{\circ} \mathrm{C}$ at a rate of $2 \mathrm{~K} / \mathrm{h}$. The excess $\mathrm{Bi}$ flux was removed by spinning

the tube in a centrifuge at $650^{\circ} \mathrm{C}$. After the centrifugation process, most of the flux contamination was removed from crystal surfaces and the remaining topical flux was etched by diluted hydrochloric acid. As shown in Fig. 1(c), the as-grown crystals were typically $1.5 \times 1.2 \times 0.8 \mathrm{~mm}^{3}$ with mirrorlike surfaces and were robust in air. As shown in Fig. 1(d), the orientation of the as-grown crystals was first checked by x-ray diffraction and the sharp (111) and (222) reflections indicate that the crystal is oriented along the [111] axis. In addition, the inset of Fig. 1(d) shows the Laue diffraction pattern of single-crystal LuPtBi generated with the beam axis coincident with the [111] zone axis. Apparently well-developed (111) planes can be observed, suggesting that our single crystals are of high quality.

The composition of all well-polished single crystals was determined by energy dispersive x-ray (EDX) spectroscopy a Hitachi S-4800 scanning electron microscope (SEM). EDX measurements were performed at different positions on the crystal surfaces within an instrument error of $1 \%-2 \%$. EDX analyses were performed at numbered points of the samples and the results are listed in Fig. S1 and Table S1, respectively, in the Supplemental Material [30]. No evidence of a Bi thin film or nanocluster was observed. According to element mapping, as shown in Fig. 2(a), Lu, Pt, and Bi are uniformly distributed across the surface, confirming the absence of a $\mathrm{Bi}$ thin film or cluster. The powder x-ray diffraction (XRD) measurements of pulverized crystals showed very sharp and strong peaks, which can all be indexed to a $\mathrm{MgAgAs}$-type structure $(F \overline{4} 3 m$ space group) [see the inset of Fig. 2(b)]. The lattice constant was calculated as $a=6.586 \AA$, which is in agreement with previous studies [31].

As shown in Fig. 3(a), the cross-sectional cuts of a selected LuPtBi crystal for scanning transmission electron microscope (STEM) measurements were prepared using a dual-beam focused ion beam (FIB) along the [110] zone axis. An aberrationcorrected STEM measurement was performed using a JEOL 2100F (JEOL, Tokyo, Japan) transmission electron microscope operated at $200 \mathrm{keV}$. The microscope was equipped with a CEOS (CEOS, Heidelberg, Germany) probe aberration (a)

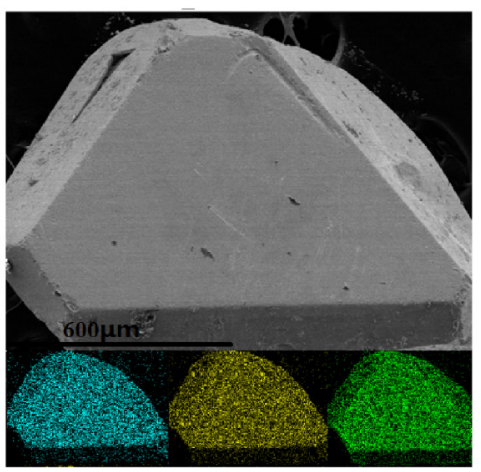

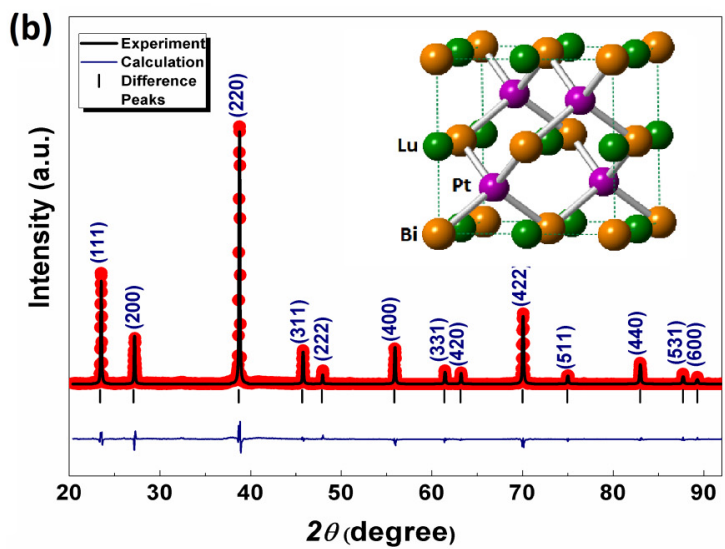

FIG. 2. (Color online) (a) A SEM image of a typical single crystal of LuPtBi after removing excess Bi flux; bottom plane. The color maps are elemental distributions of Lu (blue), Pt (yellow), and Bi (green) acquired by scanning EDX. (b) Observed (red line) powder XRD patterns of crushed LuPtBi single crystals at room temperature show the results of structural refinement (green circles). The pink segments indicate the expected diffraction peaks. LuPtBi reflections are indexed within the MgAgAs-type structure (space group $F \overline{4} 3 m, 216$ ) and the refined lattice parameter is $a=6.5861 \AA$. The inset shows a structural view of the conventional LuPtBi unit cell, with four formula units. 
(a)

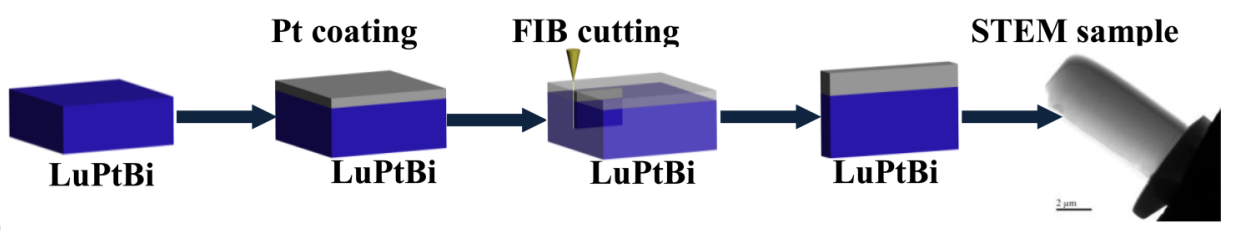

(b)
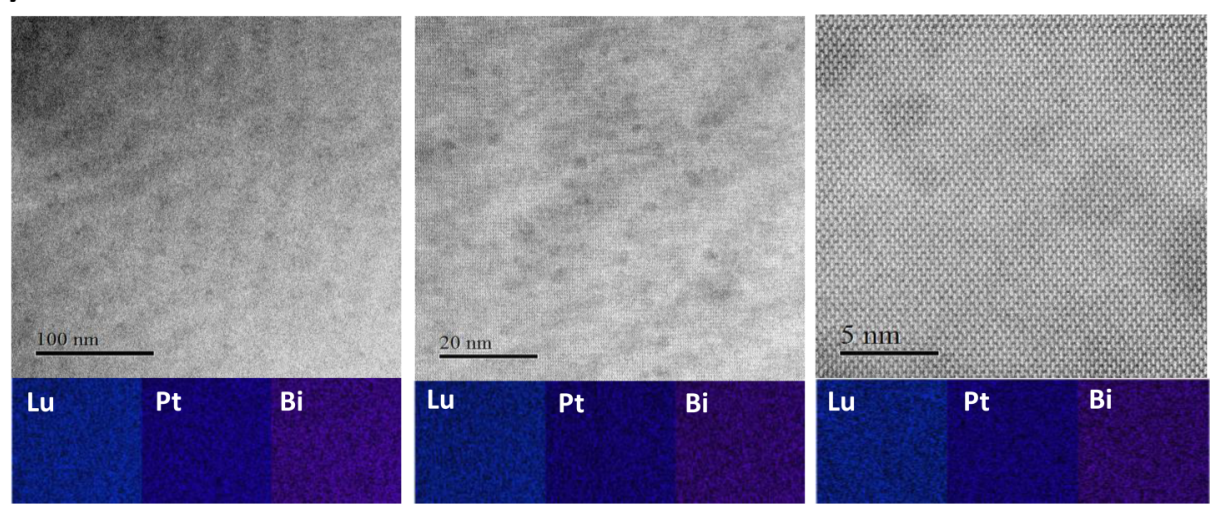

(c)

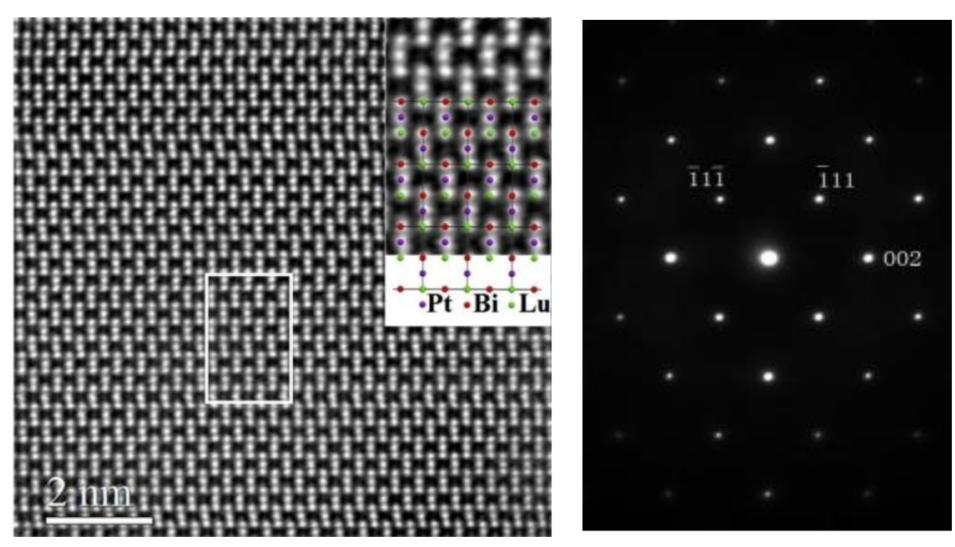

FIG. 3. (Color online) (a) The schematic for the focused ion beam (FIB) process along the [110] zone axis. (b) Top plane: STEM images at different scales along the [100] stacking axis, where no evidence of Bi thin layers or nanoclusters was observed; Bottom plane: Elemental maps of Lu, Pt, and Bi acquired by scanning EDX, demonstrating the uniform distribution of Lu, Pt, and Bi across the surface. (c) The atomic-resolution STEM image along the [100] stacking axis and selected diffraction patterns from the same plate, confirming the absence of Bi impurities.

corrector. The attainable spatial resolution of this microscope is $90 \mathrm{pm}$ at an incident semiangle of $20 \mathrm{mrad}$. As shown in Figs. 3(b) and 3(c), electron diffraction patterns and differently scaled atomically resolved dark-field scanning transmission electron microscopy (ADF-STEM) images along the [100] stacking axis, taken from many fragments of LuPtBi, showed no sign of stacking faults, intergrowths, or amorphous regions, indicating that crystal quality is good even at the nanoscale. None of the diffraction experiments indicated the presence of long- or short-range ordering of bismuth in crystals.

Electrical leads in the four-probe and Hall bar configurations were attached onto the samples using silver paste by platinum wires. Transport and specific-heat measurements were performed between 2 and $300 \mathrm{~K}$ on a Quantum Design physical properties measurement system (PPMS). More than 20 LuPtBi crystals were selected and then polished to a near-rectangular shape of size $1.5 \times 0.5 \mathrm{~mm}^{2}$ in the (111) plane and a roughly $0.2 \mathrm{~mm}$ thickness for magnetotransport measurements. Table S2 and S3 in the Supplemental Material list the transport parameters measured in six representative samples [30]. A conventional four-probe method was used for both out-of-plane resistivity and Hall measurements from 2 to $300 \mathrm{~K}$ in a magnetic field up to $14 \mathrm{~T}$ with a commercial apparatus (Quantum Design, model PPMS). The band structure calculations used the full-potential linear-augmented plane wave code implemented in the WIEN2K package including spin-orbit coupling [32].

\section{RESULTS AND DISCUSSION}

\section{A. Temperature and magnetic field dependence of resistivity}

Figure 4(a) shows the $T$-dependent resistivity $\rho_{x x}$ for the six representative samples measured at a zero field. Above $50 \mathrm{~K}$, the $\rho_{x x}-T$ curves of all samples are similar. However, as $T$ decreases from 50 to $2 \mathrm{~K}$, the values of $\rho_{x x}$ for $\mathrm{S} 1$ and S3 decrease rapidly, implying a strong enhancement of metallicity and crystal quality as well. This enhancement can be further confirmed by examining the residual resistivity ratio $\operatorname{RRR}\left[\operatorname{RRR}=\rho_{x x}(300 \mathrm{~K}) / \rho_{x x}(2 \mathrm{~K})\right]$. As shown in Fig. 4(a), the sample $\mathrm{S} 1$ had the highest value of $\mathrm{RRR}=4.2$, indicating 

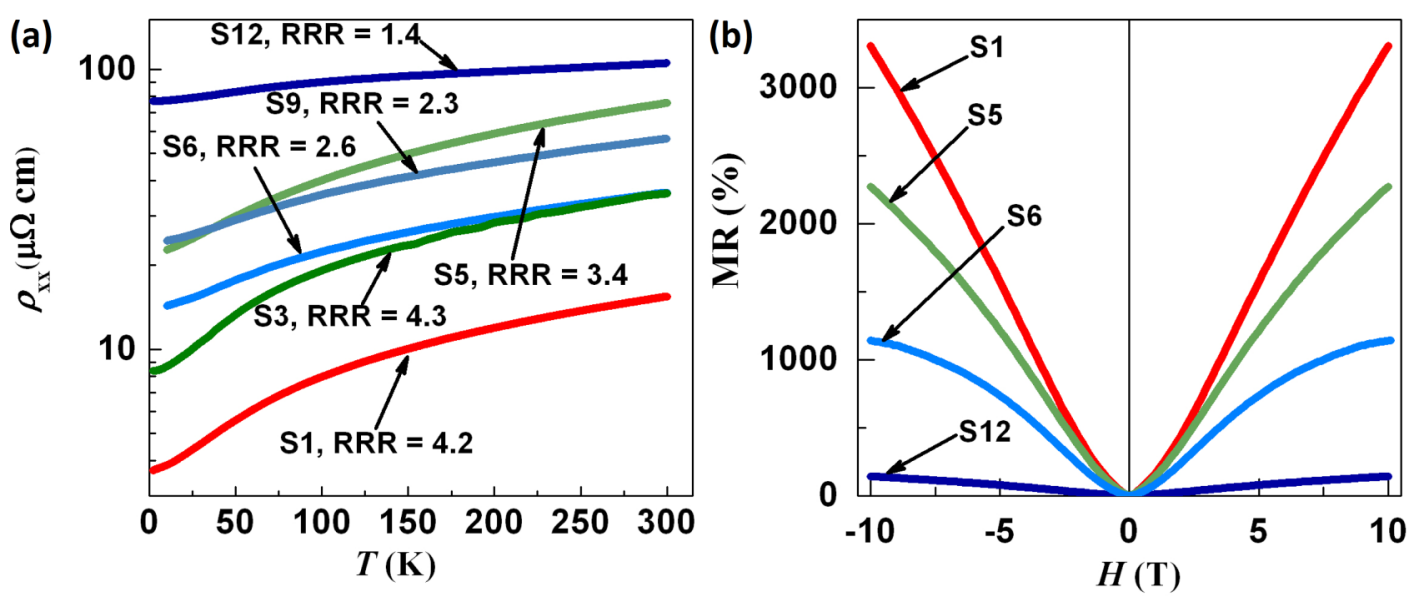

FIG. 4. (Color online) (a) Temperature dependence of zero-field resistivity $\rho_{x x}$ curves for six representative crystals with various RRRs. (b) MR curves for samples S1, S5, S6, and S12 measured at $2 \mathrm{~K}$.

a very low residual resistivity $\rho_{x x}(2 \mathrm{~K})=3 \mu \Omega \mathrm{cm}$, while for the sample $\mathrm{S} 12 R=1.4$ and therefore it has the highest residual resistivity $\rho_{x x}(2 \mathrm{~K})=78 \mu \Omega \mathrm{cm}$. As shown in Fig. 4(b), from the MR data at $2 \mathrm{~K}$, we were able to determine that the residual resistivity and $\mathrm{MR}$ are closely related to the residual resistivity ratio (RRR). As the RRR value, MR roughly increases; however, the residual resistivity decreases. In general, the RRR value is a direct measure of the defect concentration in single-crystal samples. Therefore, we can conclude that single-crystal quality is an important determinant of half-Heusler LuPtBi transport properties.

In Fig. 5(a), we show the $T$-dependent $\rho_{x x}$ for $\mathrm{S} 1$ measured at a series of applied magnetic fields. Interestingly, the applied fields change not only the $T$-dependent behavior but also significantly enhance its resistivity, especially at low temperatures. When the field is higher than $2 \mathrm{~T}$, the material begins to demonstrate semiconductinglike resistivity behavior [33]. At $2 \mathrm{~K}$, the residual resistivity increases more than 30 times (from 3.7 to $120 \mu \Omega \mathrm{cm}$ ) as the field increases from 0 to $10 \mathrm{~T}$. We propose that the quality of the crystals is the key for understanding the large sample-to-sample variation of the resistivity.
Figure 5(b) shows the MR measured at different $T$ for $\mathrm{S} 1$ in magnetic fields up to $10 \mathrm{~T}$. The MR value is defined as $[\rho(H)-\rho(0)] / \rho(0)] \times 100 \%$, where $\rho(H)$ and $\rho(0)$ are the resistivities at field $H$ and at zero field, respectively. A nonsaturating positive MR, as large as $3200 \%$ was obtained at $2 \mathrm{~K}$ and $H=10 \mathrm{~T}$. When we rotate the magnetic field from the [111] to the [1ㅣㅣ direction, the positive MR decreases to $2200 \%$ at $H=10 \mathrm{~T}$ (see Fig. S2), indicating a much smaller transport anisotropy than in layered materials, such as $\mathrm{PdCoO}_{2}$ [8] and $\mathrm{WTe}_{2}$ [9]. We note that, although at $H=10 \mathrm{~T}$ in the [111] direction, the MR is significantly suppressed by thermal fluctuation with temperature increasing from 2 to $300 \mathrm{~K}$, it is still a considerable $260 \%$. This value is comparable with that of the compensated semimetal $\mathrm{Bi}[4,34]$ but much larger than that of layered $\mathrm{PdCoO}_{2}$ [8] or $\mathrm{WTe}_{2}$ [9].

Figure 5(c) presents the so-called Kohler plot [35], in which $\Delta \rho_{x x}(H) / \rho_{x x}(0)$ is plotted as a function of $\mu_{0} H / \rho_{x x}(0)$. It is evident that the data obtained at high temperatures and low fields can be well fitted to the $H^{1.5}$ dependence expected for an orbital motion of conduction charge carriers instead of to Kohler's rule. This behavior suggests the coexistence of two
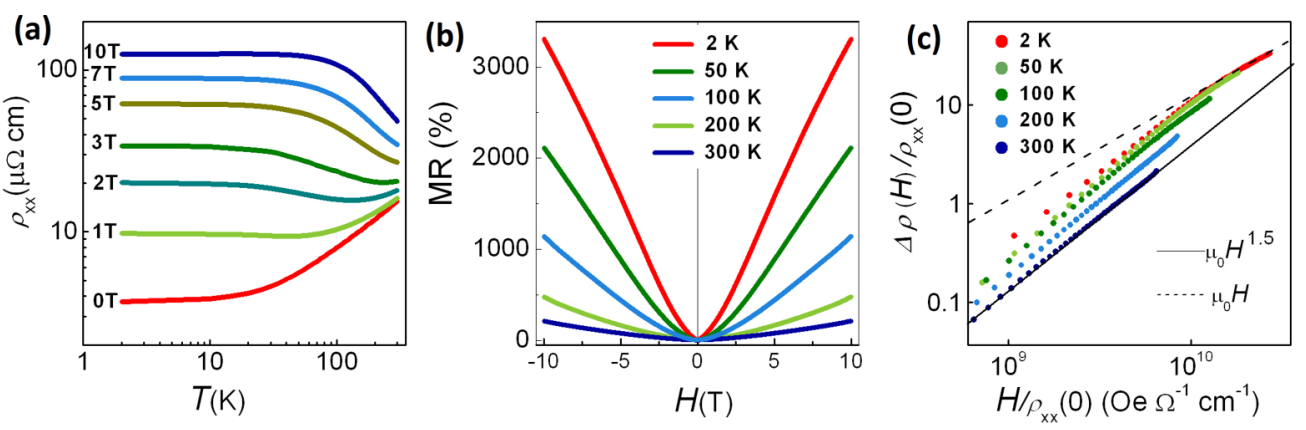

FIG. 5. (Color online) (a) Temperature dependence of the resistivity $\left(\rho_{x x}\right)$ of sample $\mathrm{S} 1$ at magnetic fields ranging from 0 to $10 \mathrm{~T}$. At $0 \mathrm{~T}$, $\rho_{x x}$ exhibits a metallic behavior. The external magnetic field increases the resistivity and changes the temperature-dependent behavior. (b) The magnetic field dependence of $\rho_{x x}$ at different temperatures. The MR ratio $\left\{\left[\rho_{x x}(H)-\rho_{x x}(0)\right] / \rho_{x x}(0)\right\}$ increases monotonically with the increase of the external magnetic field, without a saturation up to $10 \mathrm{~T}$. High MR ratios of $3200 \%$ and $260 \%$ are obtained at 2 and $300 \mathrm{~K}$, respectively. (c) The Kohler plot: the MR ratio as a function of $H / \rho_{x x}(0)$. The solid and dashed lines are the fitted lines with the equations $a H[1.5]$ and $b H$, respectively. The MR data can be scaled by the equation $a H[1.5]$ at high temperatures and in the very low-field regime at low temperatures below $50 \mathrm{~K}$ but completely deviate from Kohler's rule. At low temperatures and high-field regime, all the MR data collapse onto a single universal curve scaled linearly with $H$. 
kinds of carriers with different mobilities in the sample. On the other hand, all high field data measured at low-temperatures collapse onto a single curve that scales linearly with $H$, behavior described by Kohler's rule (see Fig. S3), which implies that at low temperatures and high field only one type of carrier dominates the electrical transport properties in this compound.

\section{B. Hall resistivity and thermoelectric power}

To gain a further insight into the carrier transport in LuPtBi, we performed Hall effect and thermoelectric power measurements. Figure 6(a) shows the magnetic field dependence of the Hall resistivity $\rho_{x y}$ at different temperatures. We note that, at all the temperatures, the slope of $\rho_{x y}$ is negative at low fields, but it changes to positive in high fields [see the enlarged figure in the inset of Fig. 6(a)]. The negative slope of $\rho_{x y}$ indicates the predominance of the electron carrier contribution to the transport at low fields; however, hole carriers take over the role when the field is large enough. By considering the highly nonlinear field dependence behavior of $\rho_{x y}$, e.g., the negative slope at low fields and the sign change, we can thus propose that LuPtBi has two kinds of carriers with different temperature- and field-dependent behaviors.

To confirm the above assumptions, we have further measured the temperature dependence of the thermoelectric power $(S)$ at $H=0$ and $9 \mathrm{~T}$, respectively, as shown in Fig. 6(b). The thermoelectric power data of LuPtBi are consistent, in a qualitative way, with the results obtained in the Hall measurements. On the one hand, at $H=0 \mathrm{~T}$, the value of $S$ is negative at $300 \mathrm{~K}$, signifying that the electron carriers dominate the thermoelectric transport. Upon cooling, the sign of $S$ shows a reversal at about $80 \mathrm{~K}$, suggesting the transport properties are dominated by hole carriers at $T<80 \mathrm{~K}$. On the other hand, at $H=9 \mathrm{~T}$, the value of $S$ only shows only a positive sign in the whole temperature range studied, which suggests a significant enhancement in the contribution of hole

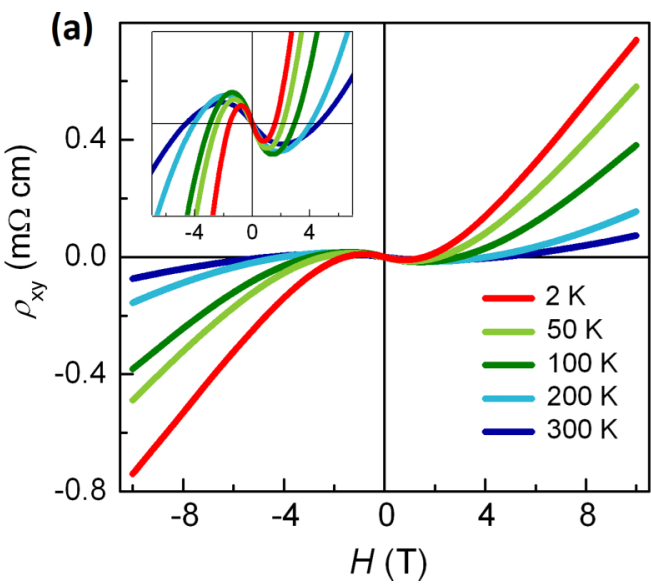

carriers at high fields, agreeing well with the Hall data. Another remarkable result is that the temperature dependences of $S$ at $H=0$ and $9 \mathrm{~T}$ are quite different, especially at $T>80 \mathrm{~K}$. In contrast to a sign reversal, $S$ at 9 T exhibits a new broad peak at $T=80 \mathrm{~K}$. Further discussion of the sign anomaly is given below. We argue that the sign changes in the Hall and $S$ signals at high fields can be related to a field-induced depopulation of electron pockets and a possible Fermi surface rearrangement.

\section{Carrier mobility and concentration}

Next, we investigated the temperature-dependent role in the conductivity tensors by the two-band model [33] to determine the mobility and concentration of the electron and hole carriers, respectively. The longitudinal conductivity $\sigma_{x x}$ and Hall conductivity $\sigma_{x y}$ can be described as

$$
\begin{gathered}
\sigma_{x x}=\frac{\rho_{x x}}{\rho_{x x}^{2}+\rho_{x y}^{2}}, \\
\sigma_{x y}=\frac{-\rho_{x y}}{\left(\rho_{x x}^{2}+\rho_{x y}^{2}\right)} .
\end{gathered}
$$

Thus, in a low-field regime $(0-1.2 \mathrm{~T})$, carrier concentrations and mobilities can be independently extracted by fitting $\sigma_{x x}$ and $\sigma_{x y}$ with the two following equations:

$$
\begin{aligned}
& \sigma_{x x}(H)=\frac{n_{e} e \mu_{e}}{1+\mu_{e}^{2} H^{2}}+\frac{n_{h} e \mu_{h}}{1+\mu_{h}^{2} H^{2}}, \\
& \sigma_{x y}(H)=\frac{n_{e} e \mu_{e}^{2} H}{1+\mu_{e}^{2} H^{2}}+\frac{n_{h} e \mu_{h}^{2} H}{1+\mu_{h}^{2} H^{2}},
\end{aligned}
$$

where $n_{e}\left(n_{h}\right)$ and $\mu_{e}\left(\mu_{h}\right)$ indicate the carrier concentrations and carrier mobilities of electrons (holes), respectively. The fitting parameters are dependent on temperature $T$ but independent of the external magnetic field $H$. Using the fitting results for both the Hall conductivity $\sigma_{x y}$ [Fig. 7(a)] and the longitudinal conductivity $\sigma_{x x}$ [Fig. 7(b)], we could

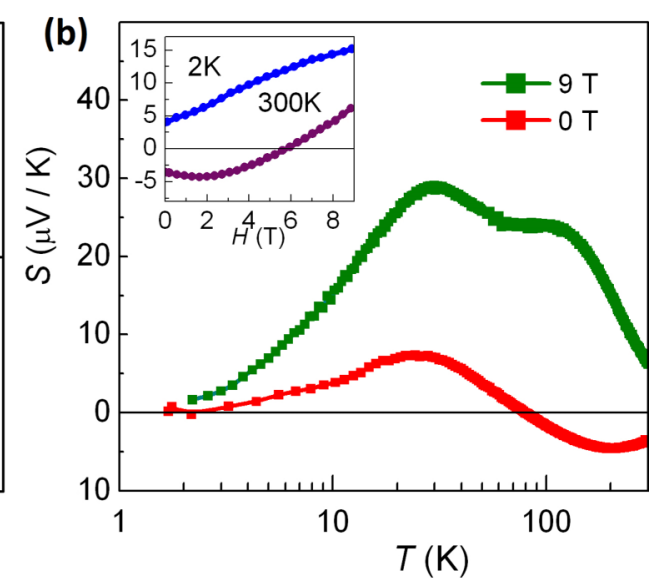

FIG. 6. (Color online) (a) The magnetic field dependence of Hall resistivity $\left(\rho_{x y}\right)$ for sample S1 at temperatures ranging from 300 to $2 \mathrm{~K}$. The inset presents the amplification of the region where $\rho_{x y}$ is close to zero and clearly exhibits the sign change in $\rho_{x y}$. At $300 \mathrm{~K}$, the negative slope of $\rho_{x y}$ indicates the predominance of electron carriers under a magnetic field below $2 \mathrm{~T}$. However, the sign changes to a positive value at higher magnetic field, indicating the further importance of hole carriers. (b) The temperature dependence of the Seebeck coefficient $(S)$ at 0 and $9 \mathrm{~T}$. The inset exhibits the magnetic field dependence of $S$ at 10 and $300 \mathrm{~K}$. At $0 \mathrm{~T}$, the Seebeck coefficient changes sign near $80 \mathrm{~K}$, confirming the two-carrier model. On the other hand, the Seebeck coefficient shows a positive value only under a magnetic field of 9 T. This indicates that the hole carrier dominates at high fields, agreeing well with Hall resistivity results. 

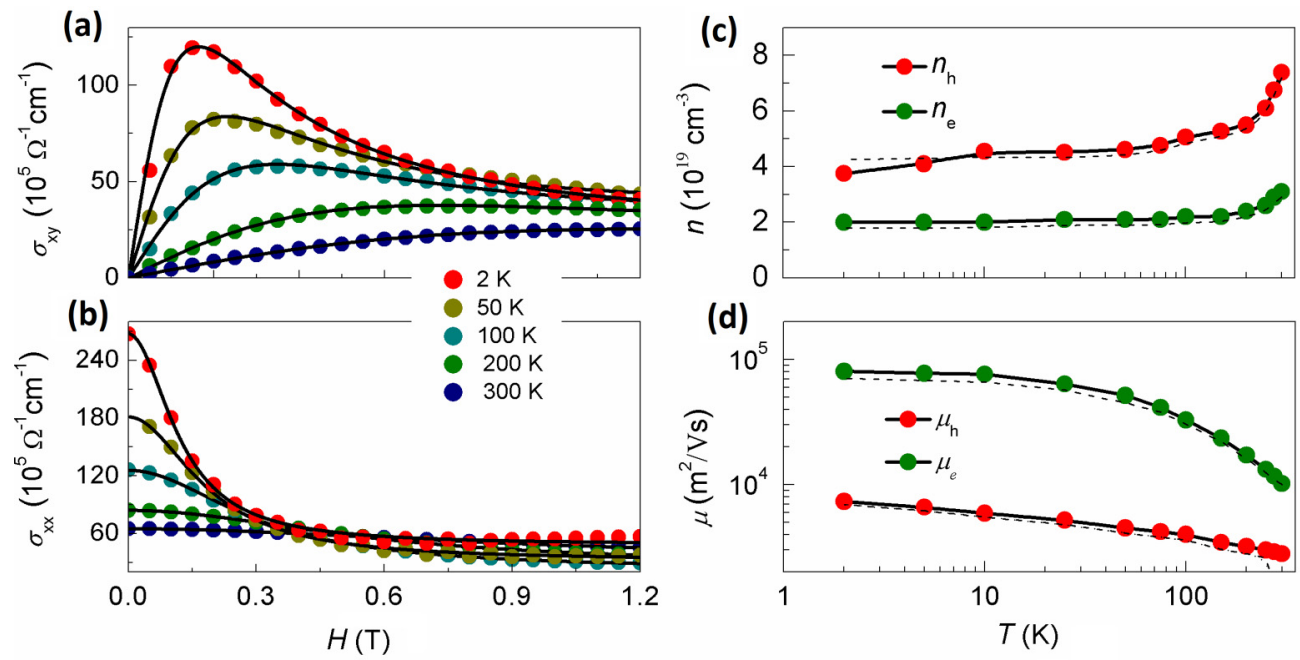

FIG. 7. (Color online) (a),(b) Magnetic field dependence of the Hall conductivity $\sigma_{x y}$ and the longitudinal conductivity $\sigma_{x x}$ for sample S1. The solid curves are the results of calculations using the two-carrier model (see the Supplemental Material [30]). (c) Temperature dependence of the electron concentration $n_{e}$ and the hole concentration $n_{h}$ estimated from the conductivity $\sigma_{x y}$. (d) Temperature dependence of the electron mobility $\mu_{e}$ and hole mobility $\mu_{h}$ estimated from the conductivity $\sigma_{x y}$.

independently obtain the Hall and longitudinal mobilities and concentrations for electron and hole carriers, respectively. As shown in Figs. 7(c) and 7(d), both the Hall and longitudinal mobilities and carrier concentrations coincide well across the entire temperature range, indicating the validity of the two-carrier model as our fitting procedure. In addition, as shown in Fig. 7(c), in the whole temperature regime, the hole carrier concentrations $n_{h}$ were slightly higher than those of electron carriers $n_{e}$, indicating that LuPtBi has a compensated nature. Notably, as shown in Fig. 7(d), the electron mobility $\mu_{e}$ is determined as $79000 \mathrm{~cm}^{2} / \mathrm{V} \mathrm{s}$ at $2 \mathrm{~K}$, which is comparable to that of the phonon glass semimetal $\beta$-CuAgSe [36]. More importantly, $\mu_{e}$ at $300 \mathrm{~K}$ is found to exceed $10500 \mathrm{~cm}^{2} / \mathrm{V} \mathrm{s}$. This appears to be the highest mobility reported at RT in any known half-Heusler semimetal and it is comparable with that of the compensated semimetal $\mathrm{Bi}$ [34] and the Dirac semimetal $\mathrm{Cd}_{3} \mathrm{As}_{2}$ [29]. In addition, the mobility of electrons is always higher than that of holes, and both values were found to be closely related to the RRR (see Fig. S4). At $2 \mathrm{~K}$, the electron-hole-compensated sample S1 had the highest mobility $\left(795000 \mathrm{~cm}^{2} / \mathrm{V} \mathrm{s}\right), \mathrm{MR}$ ratio (3200\%), and RRR, versus the hole-dominated sample S12, which had the lowest RRR, mobility $\left(1200 \mathrm{~cm}^{2} / \mathrm{V} \mathrm{s}\right)$, and MR ratio (136\%). Therefore, we suggest that the use of high-quality compensated semimetals is necessary to obtain the balance between electrons and holes that is required to achieve an ultrahigh MR, similar to that of $\mathrm{WTe}_{2}$ [9].

In Fig. 8, we compared the temperature dependence of the carrier mobility normalized by $\mu(T=2 \mathrm{~K})$ for several of the most researched high-mobility systems. The mobility of the $\mathrm{SrTiO}_{3} / \mathrm{LaAlO}_{3}$ [37] heterointerface (blue) and La-doped $\mathrm{SrTiO}_{3}$ [38] films (dark cyan) dropped rapidly with increasing temperature, while that of LuPtBi (red) exhibited only slight temperature dependence, preserving a comparatively high carrier mobility at room temperature. Such a weak temperaturedependent carrier mobility is comparable to that of the Dirac semimetal $\mathrm{Cd}_{3} \mathrm{As}_{2}$ [13], and can be quantitatively explained based on three properties of electron scattering: ionized impurity (II) scattering, acoustic phonon (AC) scattering, and optical phonon scattering (usually longitudinal, LO) [39]. By applying the form used in Ref. [39] with $\mu_{\mathrm{AC}}=$ const $\times$ $\ln \left(1+e^{E_{F} / k_{B} T}\right)$ and $\mu_{\mathrm{LO}}=$ const $\times\left(e^{\hbar \omega_{l} / k_{B} T}-1\right)$, we can reproduce the mobility changes (black line) of the Bi film (orange) [39], Ni-doped CuAgSe (green) [36], and LuPtBi (red) with varying parameters. As indicated by the dotted line, longitudinal optical phonon scattering is a primary source of carrier scattering in these materials at high temperatures

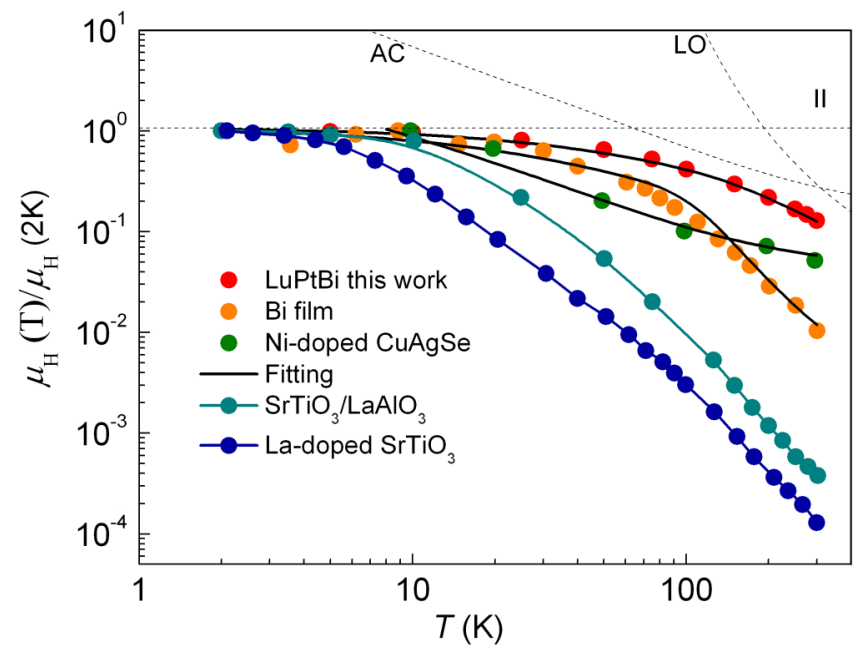

FIG. 8. (Color online) The normalized $\mu_{H}(T) / \mu_{H}(2 \mathrm{~K})$ of LuPtBi is compared with those of several of the most researched high-mobility systems. Clearly, the mobility of the $\mathrm{SrTiO}_{3} / \mathrm{LaAlO}_{3}$ interface (blue) and La-doped $\mathrm{SrTiO}_{3}$ films (dark cyan) dropped rapidly with increasing temperature, while for semimetal systems, such as the Bi film (orange), Ni-doped $\mathrm{CuAgSe}$ (green), and LuPtBi (red), the temperature dependence is weak, preserving relatively high carrier mobility at room temperature. The solid lines are the fitting curves (see text for more details). 

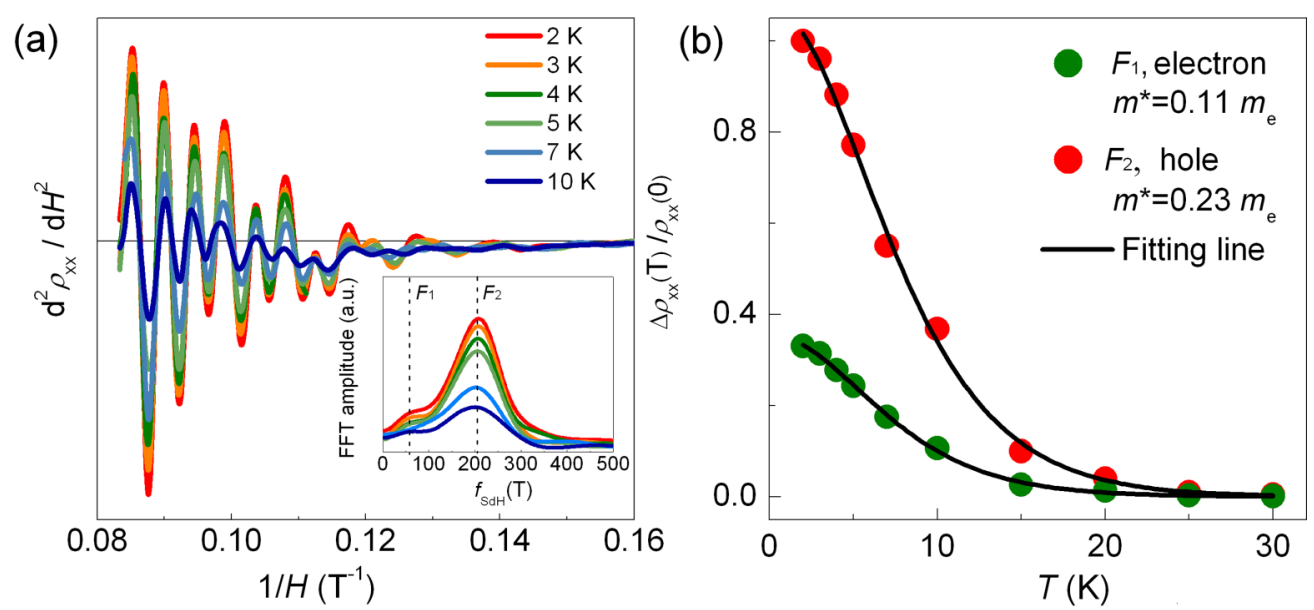

FIG. 9. (Color online) (a) Temperature dependence of the SdH oscillations $d^{2} \rho_{x x} / d H^{2}$ as a function of the inverse magnetic field $1 / H$. The inset shows the fast Fourier transform (FFT) spectra of SdH oscillations at different temperatures, in which $F_{1}$ and $F_{2}$ denote the electron and hole Fermi pockets, respectively. (b) Temperature dependence of the normalized FFT amplitude for the electron and hole Fermi pockets. The red and blue solid lines are the fits with the standard Lifshitz-Kosevich formula, yielding the small effective masses $0.11 m_{e}$ and $0.23 m_{e}$ for the electron and hole Fermi pockets, respectively.

[40]. Therefore, we assume that the weak dependence of mobility on temperature in LuPtBi can be attributed to the significant reduction in scattering from longitudinal optical phonons as a consequence of peculiarities in the band structure.

\section{Shubnikov-de Haas quantum oscillations}

To understand the electronic states that contribute to transport properties, we investigate the MR and Shubnikov-de Haas $(\mathrm{SdH})$ quantum oscillations at high fields. In Fig. S5, we show the temperature dependence of $\mathrm{SdH}$ quantum oscillations at high magnetic fields for samples S1 and S3. The field is applied parallel to the [111] direction. At high fields, the clear $\mathrm{SdH}$ oscillations are superimposed on a huge background of positive MR. In Fig. 9(a), we highlight the SdH oscillations by plotting $d^{2} \rho_{x x} / d H^{2}$ as a function of the inverse magnetic field $(1 / H)$ for sample $S 1$. As seen in the inset, prominent $\mathrm{SdH}$ oscillations become visible at $H>5 \mathrm{~T}$, indicating the extreme mobility of carriers in this sample. We confirm that $\mathrm{SdH}$ oscillations are observable when $H$ changes from $0^{\circ}$ to $90^{\circ}$, that is, from the [111] to the $1 \overline{1} 0$ direction, suggesting their 3D origin (see Fig. S6). The details of the SdH oscillations in all geometries will be discussed elsewhere. The inset of Fig. 9(a) shows the fast Fourier transform (FFT) spectra of oscillation at $0^{\circ}$, revealing two peaks at $F_{1}=80 \mathrm{~T}$ and $F_{2}=200 \mathrm{~T}$, which are confirmed as the electron and hole Fermi pockets, respectively (see Fig. S7). By using the Onsager relationship $F=(\hbar / 2 \pi e) A_{F}$, where $e$ is the electron charge, $\hbar$ is Planck's constant, and $A_{F}$ is the cross-sectional area of the Fermi surface normal to the magnetic fields, we found the sizes of the electron and hole Fermi pockets to be $0.01 \AA^{-2}$ and $0.019 \AA^{-2}$, respectively. The corresponding Fermi wave vectors $\kappa_{F}$ for the electron and hole Fermi pockets are $0.048 \AA^{-2}$ and $0.086 \AA^{-2}$, respectively. According to the standard Lifshitz-Kosevich theory [41], the cyclotron effective mass of the carriers $\left(m^{*}\right)$ can be obtained by fitting the temperature dependence of the normalized FFT amplitudes with a thermal damping factor

$$
R_{T}=\frac{2 \pi^{2} \kappa_{B} T / \hbar \omega_{c}}{\sinh \left[2 \pi^{2} \kappa_{B} T / \hbar \omega_{c}\right]},
$$

where $\kappa_{B}$ is Boltzmann's constant, $T$ is the temperature and, $\omega_{c}=e H / m^{*}$ is the cyclotron frequency, which directly results in the effective mass $m^{*}$. As shown in Fig. 9(b), the effective masses for the electron and hole Fermi pockets yielded by the fits are $0.11 m_{e}$ and $0.23 m_{e}\left(m_{e}\right.$ is the free electron mass), respectively. Finally, the Fermi velocities for the electron and hole pockets are calculated using $v_{F}=\hbar k_{F} / m^{*}$; they are $5.5 \times$ $10^{7} \mathrm{~cm} \mathrm{~s}^{-1}$ and $3.3 \times 10^{7} \mathrm{~cm} \mathrm{~s}^{-1}$, respectively. Both values are close to those of the Dirac semimetal $\mathrm{Cd}_{3} \mathrm{As}_{2}[29,42]$ and the Weyle semimetals TaAs [43] and NbP [44]. Thus, the large Fermi velocity and small effective mass are responsible for the observed ultrahigh mobility in LuPtBi. Note that although the quantum limit is actually not reached in our LuPtBi crystal, there is clearly more than one Landau level occupied in our field range. Therefore, the appearance of a linear MR without reaching the quantum limit requires further investigation.

\section{E. Band structure and Fermi surface}

Band structure calculations provide insight into the origin of an electronic structure with ultrahigh mobility and MR such as in LuPtBi. As shown in Fig. 10(a), the profile of the density of states (DOS) reveals that the Fermi level $\left(E_{F}\right)$ of LuPtBi is located at the valley of the DOS, confirming that the states at the Fermi level are dominated by the states of Pt and Bi atoms. Our band structure calculations are illustrated in Fig. 10(b), and as in a previous report [45], the valence and conduction bands barely cross the $E_{F}$ and do so at different places, indicating that LuPtBi is an electron-hole-compensated semimetal. This can also be confirmed by the volume of the Fermi electron and hole pockets showing in Fig. 10(c), which implies Fermi surface compensation in LuPtBi. The Fermi surface of LuPtBi consists of eight equivalent needlelike electron pockets and one hole pocket located almost isotropically at the center of the 
(a)

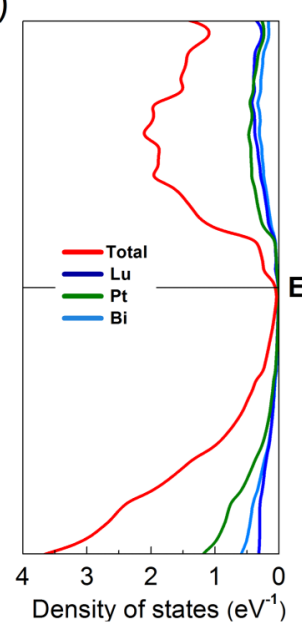

(b)

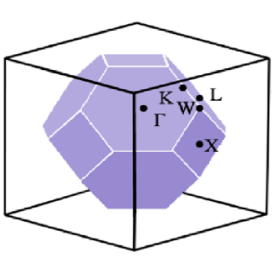

Brillouin zone
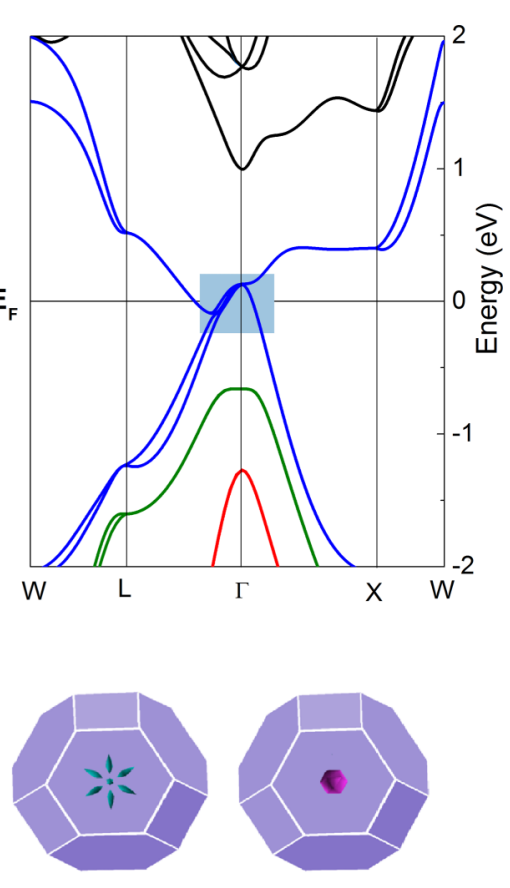

Electron pocket

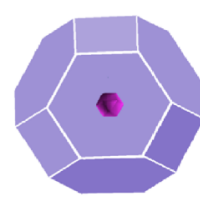

Hole pocket

FIG. 10. (Color online) (a) The total density of states (DOS) and local DOS from $\mathrm{Lu}, \mathrm{Pt}$, and $\mathrm{Bi}$ in the LuPtBi complex. The main peaks of the total DOS are located far from the Fermi level and $\mathrm{Lu}$, $\mathrm{Pt}$, and Bi contribute equally to the total DOS. (b) The calculated band structure of LuPtBi. The black line at $E=0$ shows the Fermi level position. The coexistence of electron and hole pockets agrees well with the two-carrier analysis. (c) The bulk Brillouin zone (left plane) and the Fermi surface of bulk LuPtBi showing electron (middle plane) and hole (right plane) pockets.

Brillouin zone along the crystallographic [111] directions; for each crystallographic [111] direction, two needlelike electron pockets are located symmetrically with respect to the $\Gamma$ point, and the angle of the two adjacent ellipsoid pockets is $70.52^{\circ}$. At the crystallographic (111) plane, six equivalent needlelike electron pockets are located symmetrically around the [111] axis and each electron pocket can be obtained by a $60^{\circ}$ rotation around the [111] axis. This exceptional feature of the electronic structure is reminiscent of those of the well-known semimetal $\mathrm{Bi}[10,33]$ and the layered alloy $\mathrm{NbSb}_{2}[46]$. The unique shape of the Fermi surface of LuPtBi crystals is energetically unfavorable; therefore, a magnetic field can cause the contacting $p$-symmetry band to split such that a band gap appears at the Fermi level, which may cause the carrier concentration and mobility tensor changes with magnetic field. On the other hand, the highly anisotropic electron pockets may cause large variations in mobility, which may also help to explain its unusual large positive MR. In this regard, further angle-resolved photoemission spectroscopy experiments are necessary to verify the Fermi surface topology and its evolution with $H$ from which the large nonsaturating linear MR may originate.

The next question to be addressed is why the signs of the Hall resistivity and thermoelectric power data change in high fields. In fact, the sign change of the Hall resistivity with $H$ occurs only when the two kinds of carriers have distinct mobility and/or concentration. In our LuPtBi sample, the high-

mobility electrons with a small effective mass dominate the electrical transport and gives rise to the negative Hall resistivity in low-fields. In order to achieve the sign reversal of the Hall resistivity and thermoelectric power in high fields, the number of high-mobility electrons should be much smaller than the number of low-mobility holes. In this case, the sign change is reminiscent of the change of the Fermi surface topology (field-induced Lifshitz transition [47]) in momentum space in gapless materials, such as HgTe [48] and the half-Heusler CePtBi [49]. The scenario is that the small Fermi surface is energetically unfavorable in high fields. Therefore, the magnetic field may cause the displacement of the energy band edges, which may further induce the contacting $p$-symmetry band split, and as a result, the concentration of high-mobility electrons will decrease while the concentration of low-mobility holes will increase instead. Further study will be required to verify this.

\section{CONCLUSION}

We end by summarizing the important features of the half-Heusler LuPtBi semimetal. First, it shows an extremely high electron mobility up to $79000 \mathrm{~cm}^{2} / \mathrm{V} \mathrm{s}$ together with a nonsaturating positive MR as large as $3200 \%$ and at $2 \mathrm{~K}$. Remarkably, the electron mobility at $300 \mathrm{~K}$ is found to exceed $10500 \mathrm{~cm}^{2} / \mathrm{V} \mathrm{s}$, which appears to be among the highest values reported in 3D bulk materials thus far. Second, it has a distinctive compensated band structure showing a rather small effective carrier mass as derived from the $\mathrm{SdH}$ quantum oscillations. The small effective carrier mass is responsible for the extremely high electron mobility and large positive MR. Third, its high-mobility electron conduction is robust against thermal fluctuation, as evidenced by the weak temperature-dependent electron mobility that results from a significant reduction in scattering from longitudinal optical phonons. Last, the electron-hole-compensated band structure is rather sensitive to the applied magnetic fields, as evidenced by the observation of the signs of the Hall resistivity and thermoelectric power in high fields. However, it remains to be investigated how and why the fields can influence the Fermi surface topology. Nevertheless, our results indicate that, even in the reported 3D semimetals, both ultrahigh mobility and large positive MR may be obtained by the appropriate design of their band structure with the use of the Fermi surface compensated strategy. We expect that the strategy will provide useful guidelines for the development of ultrahigh-carrier-mobility semimetals showing large positive MR as high-performance magnetoresistive devices in other known half-Heusler semimetals and open an area of research of both fundamental and applied importance.

\section{ACKNOWLEDGMENTS}

We thank Professor J. R. Sun and Professor C. Felser for fruitful discussions. This work is supported by the National Basic Research Program of China (973 Program Grant No. 2012CB619405), the National Natural Science Foundation of China (Grants No. 51171207 and No. 11474343), and Strategic Priority Research Program B of the Chinese Academy of Sciences under Grant No. XDB07010300. 
[1] M. N. Baibich, J. M. Broto, A. Fert, F. Nguyen Van Dau, F. Petroff, P. Etienne, G. Creuzet, A. Friederich, and J. Chazelas, Phys. Rev. Lett. 61, 2472 (1988).

[2] A. E. Berkowitz, J. R. Mitchell, M. J. Carey, A. P. Young, S. Zhang, F. E. Spada, F. T. Parker, A. Hutten, and G. Thomas, Phys. Rev. Lett. 68, 3745 (1992).

[3] J. Q. Xiao, J. S. Jiang, and C. L. Chien, Phys. Rev. Lett. 68, 3749 (1992).

[4] R. E. Camley, and R. L. Stamps, J. Phys.: Condens. Matter 5, 3727 (1993).

[5] S. Jin, T. H. Tiefel, M. Mccormack, R. A. Fastnacht, R. Ramesh, and L. H. Chen, Science 264, 413 (1994).

[6] Y. Tokura, Colossal Magnetoresistive Oxides, (Gordon and Breach Science, Amsterdam, Netherlands, 2000).

[7] R. Xu, A. Husmann, T. F. Rosenbaum, M. -L. Saboungi, J. E. Enderby, and P. B. Littlewood, Nature (London) 390, 57 (1997).

[8] M. Lee, T. F. Rosenbaum, M. -L. Saboungi, and H. S. Schnyders, Phys. Rev. Lett. 88, 066602 (2002).

[9] J. Hu, and T. F. Rosenbaum, Nat. Mater. 7, 697 (2008).

[10] F. Y. Yang, K. Liu, K. M. Hong, D. H. Reich, P. C. Searson, and C. L. Chien, Science 284, 1335 (1999).

[11] A. L. Friedman, J. L. Tedesco, P. M. Campbell, J. C. Culbertson, E. Aifer, F. K. Perkins, R. L. Myers-Ward, J. K. Hite, C. R. Eddy, Jr., G. G. Jernigan, and D. K. Gaskill, Nano Lett. 10, 3962 (2010).

[12] Z. M. Liao, H. C. Wu, S. Kumar, G. S. Duesberg, Y. B. Zhou, Graham L. W. Cross, Igor V. Shvets, and D. P. Yu, Adv. Mater. 24, 1862 (2012).

[13] J. Feng, Y. Pang, D. Wu, Z. Wang, H. Weng, J. Li, X. Dai, Z. Fang, Y. G. Shi, and L. Lu, Phys. Rev. B. 92, 081306(R) (2015).

[14] H. Takatsu, J. J. Ishikawa, S. Yonezawa, H. Yoshino, T. Shishidou, T. Oguchi, K. Murata, and Y. Maeno, Phys. Rev. Lett. 111, 056601 (2013).

[15] D. X. Qu, Y. S. Hor, J. Xiong, R. J. Cava, and N. P. Ong, Science 329, 821 (2010).

[16] X. L. Wang, Y. Du, S. X. Dou, and C. Zhang, Phys. Rev. Lett. 108, 266806 (2012).

[17] M. M. Parish, and P. B. Littlewood, Nature (London) 426, 162 (2003).

[18] A. A. Abrikosov, Europhys. Lett. 49, 789 (2000).

[19] M. N. Ali, J. Xiong, S. Flynn, J. Tao, Q. D. Gibson, L. M. Schoop, T. Liang, N. Haldolaarachchige, M. Hirschberger, N. P. Ong, and R. J. Cava, Nature (London) 514, 205 (2014).

[20] I. Pletikosić, M. N. Ali, A. V. Fedorov, R. J. Cava, and T. Valla, Phys. Rev. Lett. 113, 216601 (2014).

[21] T. Graf, C. Felser, and S. S. P. Parkin, Prog. Solid State Chem. 39, 1 (2011).

[22] S. Chen, and Z. F. Ren, Mater. Today 16, 387 (2013).

[23] S. Chadov, X. L. Qi, J. Kübler, G. H. Fecher, C. Felser, and S. C. Zhang, Nat. Mater. 9, 541 (2010).

[24] H. Lin, L. A. Wray, Y. Q. Xia, S. Y. Xu, S. Jia, R. J. Cava, A. Bansil, and M. Z. Hasan, Nat. Mater. 9, 546 (2010).

[25] D. Xiao, Y. G. Yao, W. X. Feng, J. Wen, W. G. Zhu, X. Q. Chen, G. M. Stocks, and Z. Y. Zhang, Phys. Rev. Lett. 105, 096404 (2010).

[26] C. Shekhar, S. Ouardi, A. K. Nayak, G. H. Fecher, W. Schnelle, and C. Felser, Phys. Rev. B. 86, 155314 (2012).
[27] W. H. Wang, Y. Du, G. Z. Xu, X. M. Zhang, E. K. Liu, Z. Y. Liu, Y. G. Shi, J. L. Chen, G. H. Wu, and X. X. Zhang, Sci. Rep. 3, 2181 (2013).

[28] G. Z. Xu, W. H. Wang, X. M. Zhang, Y. Du, E. K. Liu, S. G. Wang, G. H. Wu, Z. Y. Liu, and X. X. Zhang, Sci. Rep. 4, 5709 (2014).

[29] T. Liang, Q. Gibson, M. N. Ali, M. Liu, R. J. Cava, and N. P. Ong, Nat. Mater. 14, 280 (2015).

[30] See Supplemental Material at http://link.aps.org/supplemental/ 10.1103/PhysRevB.92.235134 for EDX, thickness-dependent mobility, and MR data, sample-dependent SdH oscillations, and computation details.

[31] F. F. Tafti, T. Fujii, A. Juneau-Fecteau, S. René de Cotret, N. Doiron-Leyraud, A. Asamitsu, and L. Taillefer, Phys. Rev. B 87, 184504 (2013).

[32] P. Blaha, K. Schwarz, G. Madsen, D. Kvaniscka, and J. Luitz, Computer code WIEN2K, An Augmented Plane Wave Plus Local Orbitals Program for Calculating Crystal Properties (Vienna University of Technology, Vienna, Austria, 2001).

[33] N. W. Ashcroft, Solid State Physics (Holt, Rinehart and Winston, New York, 1976).

[34] X. Qu, S. W. Tsai, D. L. Maslov, and A. F. Hebard, Phys. Rev. Lett. 94, 166601 (2005).

[35] A. B. Pippard, Magnetoresistance in Metals (Cambridge University Press, Cambridge, 1989).

[36] S. Ishiwata, Y. Shiomi, J. S. Lee, M. S. Bahramy, T. Suzuki, M. Uchida, R. Arita, Y. Taguchi, and Y. Tokura, Nat. Mater. 12, 512 (2013).

[37] A. Ohtomo, and H. Y. Hwang, Nature (London) 427, 423 (2004).

[38] Amit Verma, Adam P. Kajdos, Tyler A. Cain, Susanne Stemmer, and Debdeep Jena, Phys. Rev. Lett. 112, 216601 (2014).

[39] Sunglae Cho, Yunki Kim, A. J. Freeman, G. K. L. Wong, J. B. Ketterson, L. J. Olafsen, I. Vurgaftman, J. R. Meyer, and C. A. Hoffman, Appl. Phys. Lett. 79, 3651 (2001).

[40] B. I. Shklovskii and A. L. Efros, Electronic Properties of Doped Semiconductors (Springer-Verlag, Berlin, 1984).

[41] D. Shoenberg, Magnetic Oscillations in Metals (Cambridge University Press, London, 1984).

[42] A. Narayanan, M. D. Watson, S. F. Blake, N. Bruyant, L. Drigo, Y. L. Chen, D. Prabhakaran, B. Yan, C. Felser, T. Kong, P. C. Canfied, and A. I. Coldea, Phys. Rev. Lett. 114, 117201 (2015).

[43] C. L. Zhang, Z. J. Yuan, S. Y. Xu, Z. Q. Lin, B. B. Tong, M. Z. Hasan, J. F. Wang, C. Zhang, and S. Jia, arXiv:1502.00251.

[44] C. Shekha, A. Nayak, Y. Sun, M. Schmidt, M. Nicklas, I. Leermakers, U. Zeitler, Y. Skourski, J. Wosnitza, Z. Liu, Y. Chen, W. Schnelle, H. Borrmann, Y. Grin, C. Felser, and B. Yan, Nat. Phys. 11, 645 (2015).

[45] W. Al-Sawai, H. Lin, R. S. Markiewicz, L. A. Wray, Y. Xia, S. -Y. Xu, M. Z. Hasan, and A. Bansil, Phys. Rev. B 82, 125208 (2010)

[46] K. Wang, D. Graf, L. J. Li, L. M. Wang, and C. Petrovic, Sci. Rep. 4, 7328 (2014).

[47] I. M. Lifshitz, Zh. Eksp. Teor. Fiz. 38, 1569 (1960) [Sov. Phys. JETP 11, 1130 (1960)].

[48] T. F. Rosenbaum, S. B. Field, D. A. Nelson, and P. B. Littlewood, Phys. Rev. Lett. 54, 241 (1985).

[49] N. Kozlova, J. Hagel, M. Doerr, J. Wosnitza, D. Eckert, K.-H Müller, L. Schultz, I. Opahle, S. Elgazzar, M. Richter, G. Goll, H. v. Löhneysen, G. Zwicknagl, T. Yoshino, and T. Takabatake, Phys. Rev. Lett. 95, 086403 (2005). 